\title{
MIST: Cellular Data Network Measurement for Mobile Applications
}

Mike P. Wittie, Brett Stone-Gross, Kevin C. Almeroth and Elizabeth M. Belding Department of Computer Science, University of California, Santa Barbara
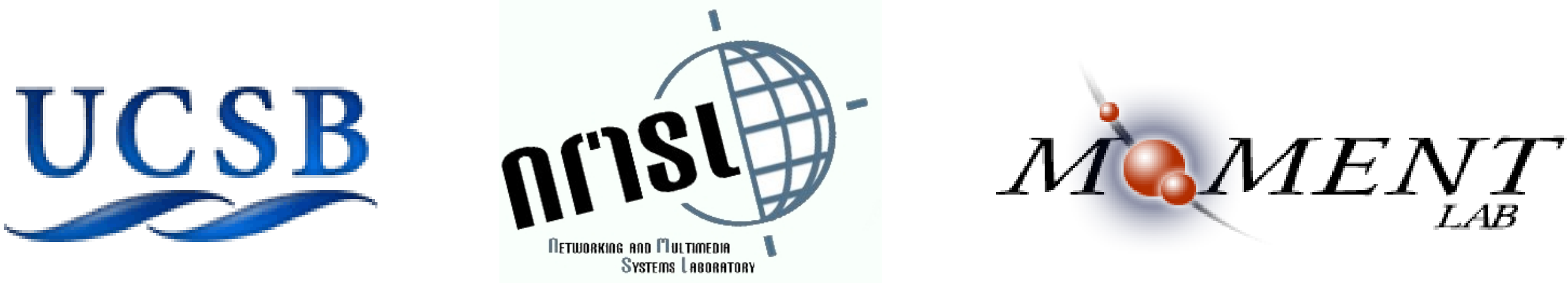


\section{Introduction}

- Cellular data network growth

- SMS, email, Internet, Video

- Mobile application developers ready to cash in

- Quick-and-quality application development

- Unknown device and network performance 


\section{Mobile Application Challenges}

\begin{tabular}{l|l|l} 
Application Type & Challenges & Optimization Techniques \\
\hline Streaming Media & -limited bandwidth & - layered encoding \\
Pervasive Gaming & - latency variation & - system specific timeouts \\
Two-way Database & - radio timeout & - keep alive packets
\end{tabular}




\section{Mobile Internet Services Test (MIST)}

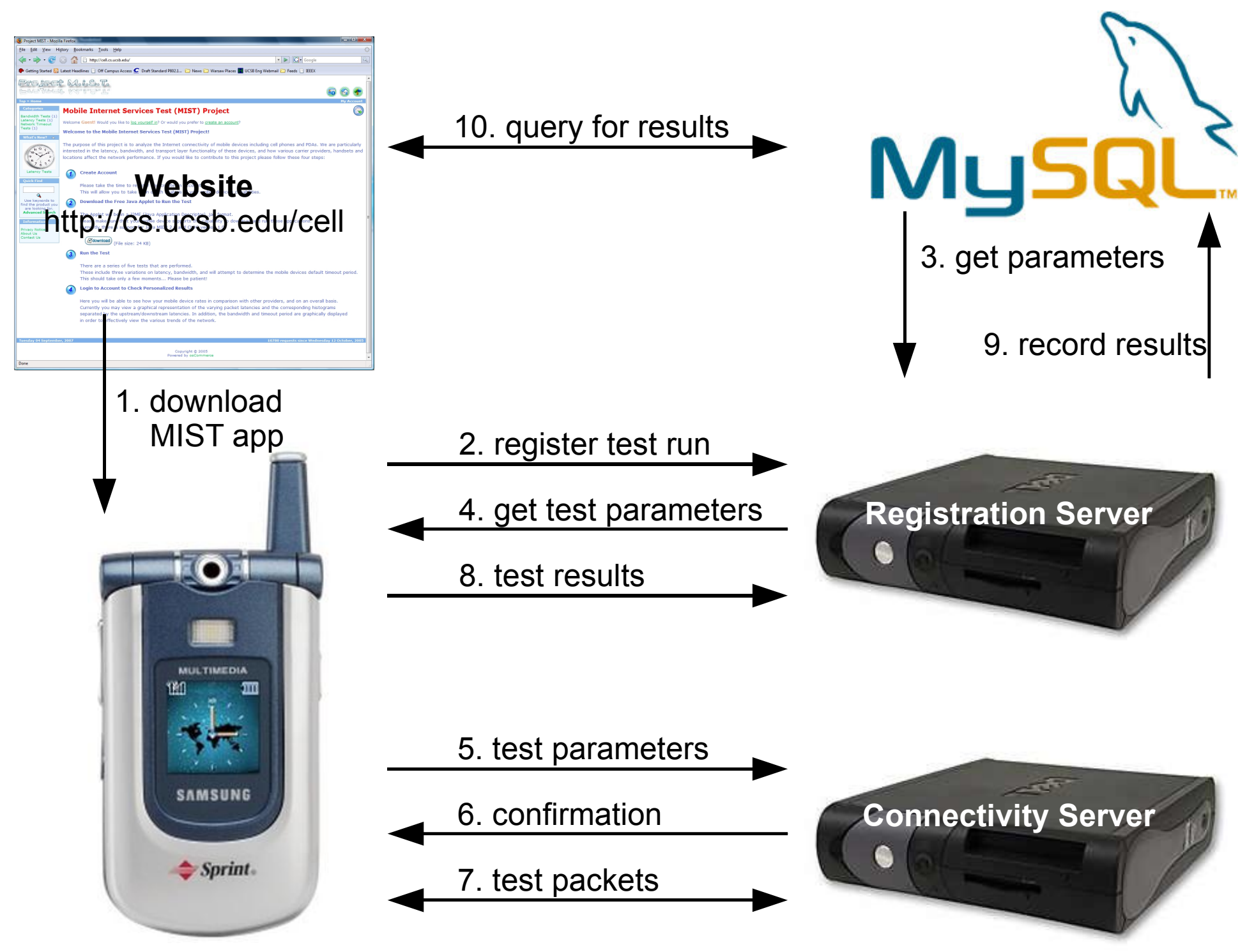




\section{MIST Network Measurement}

Latency
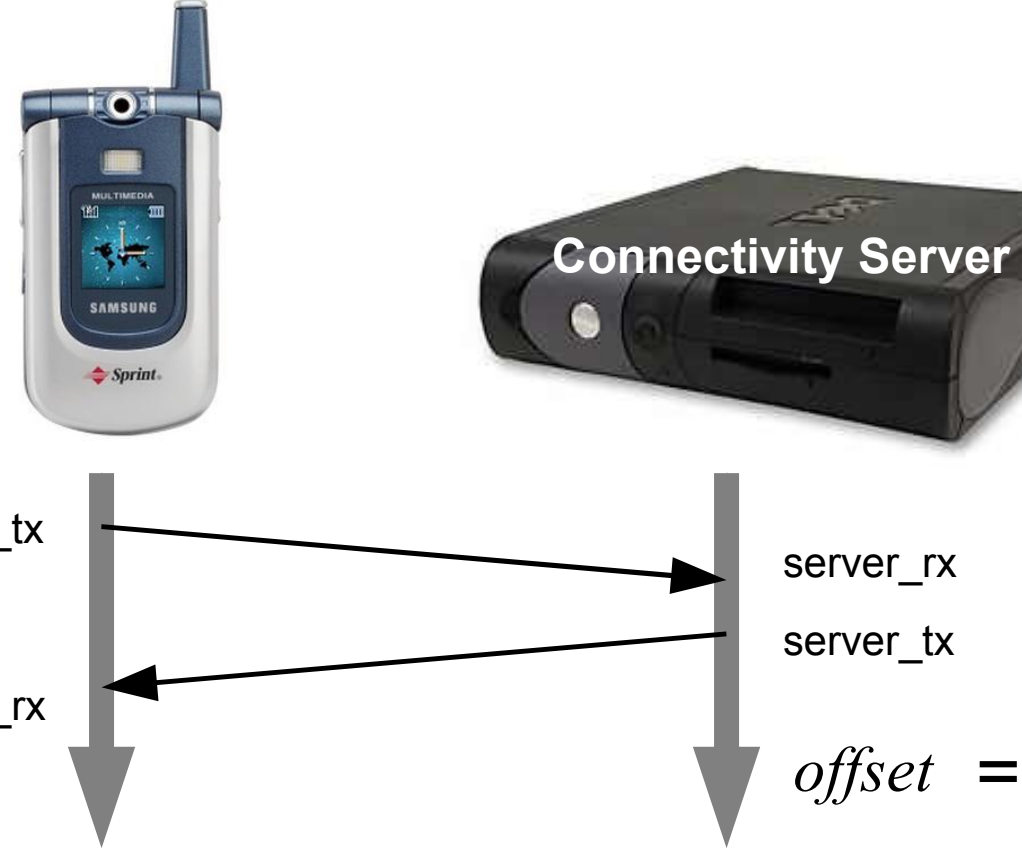

server_rx

server_tx

offset $=\frac{r x_{\text {server }}-t x_{\text {client }}+t x_{\text {server }}-r x_{\text {client }}}{2}$

Throughput

Timeout Interval
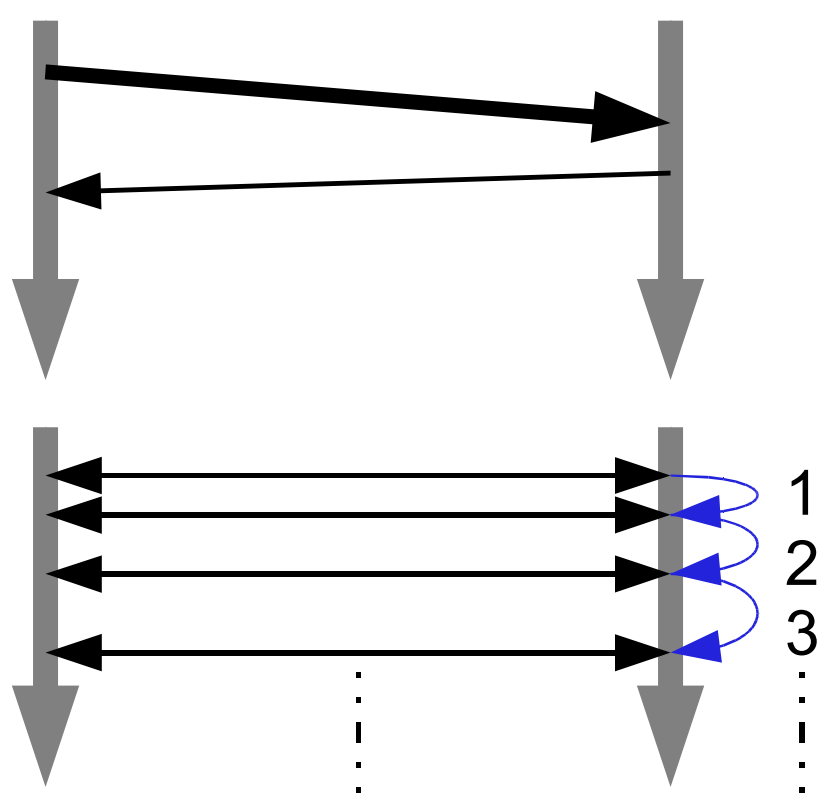


\section{Uplink and Downlink Latency}

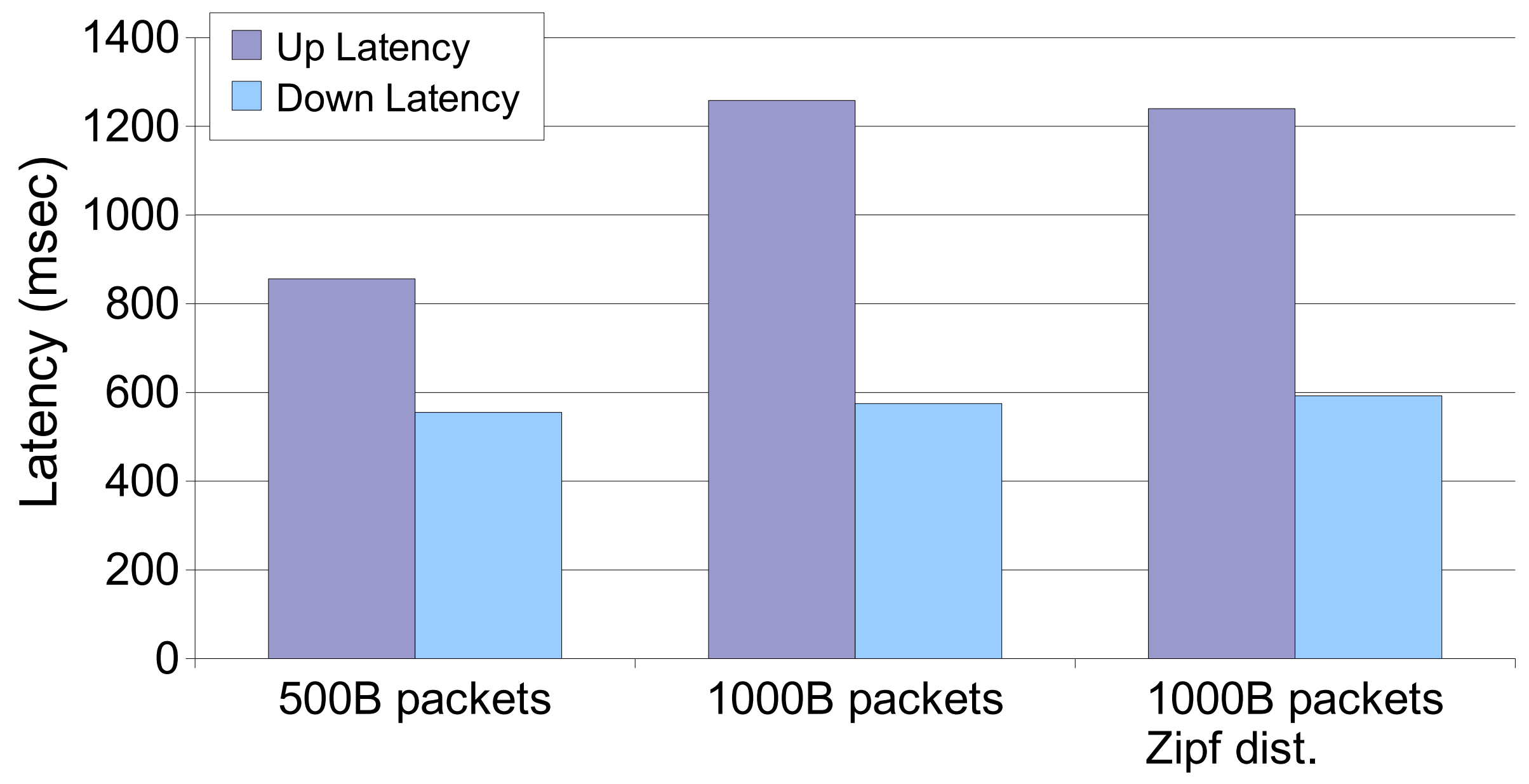




\section{Latency and Mobility}

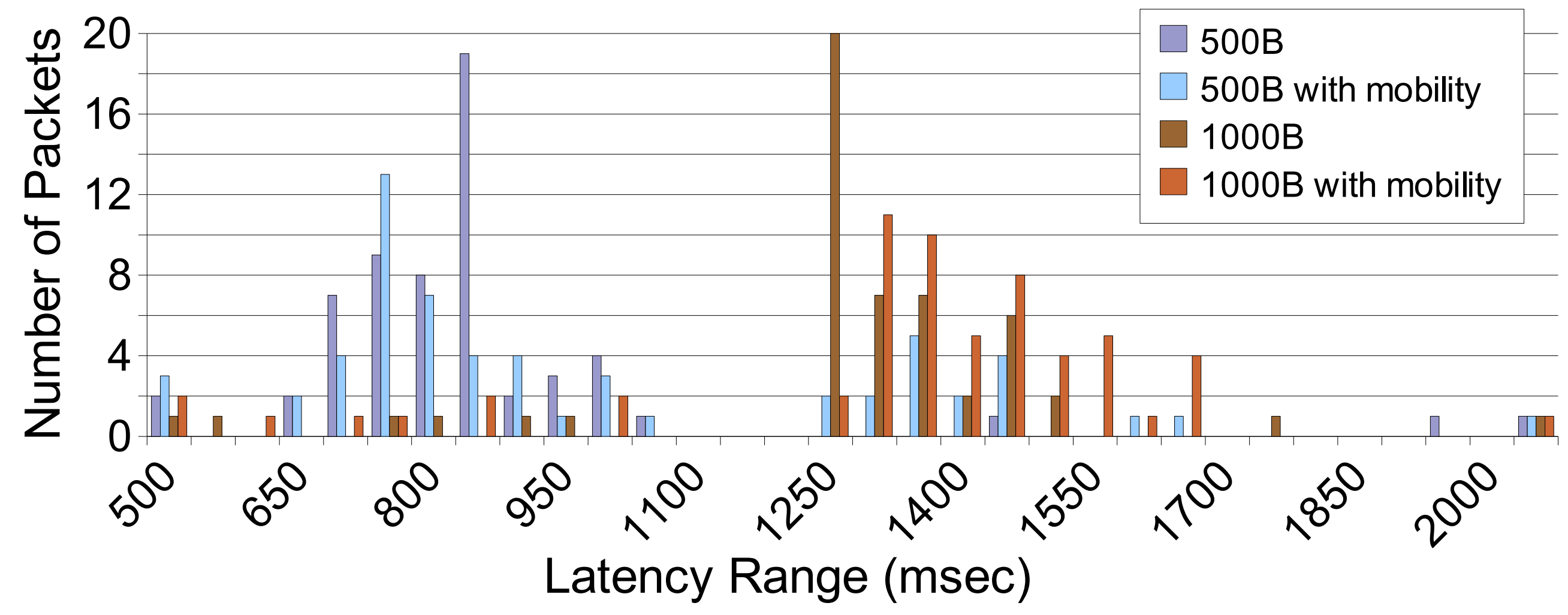




\section{Service Throughput}

\begin{tabular}{l|r|r}
\hline Throughput (KBPS) & \multicolumn{1}{|c|}{ Uplink } & \multicolumn{1}{c}{ Downlink } \\
\hline Minimum & 0.79 & 1.82 \\
\hline Average & 2.05 & 4.67 \\
\hline Maximum & 4.47 & 11.17 \\
\hline
\end{tabular}

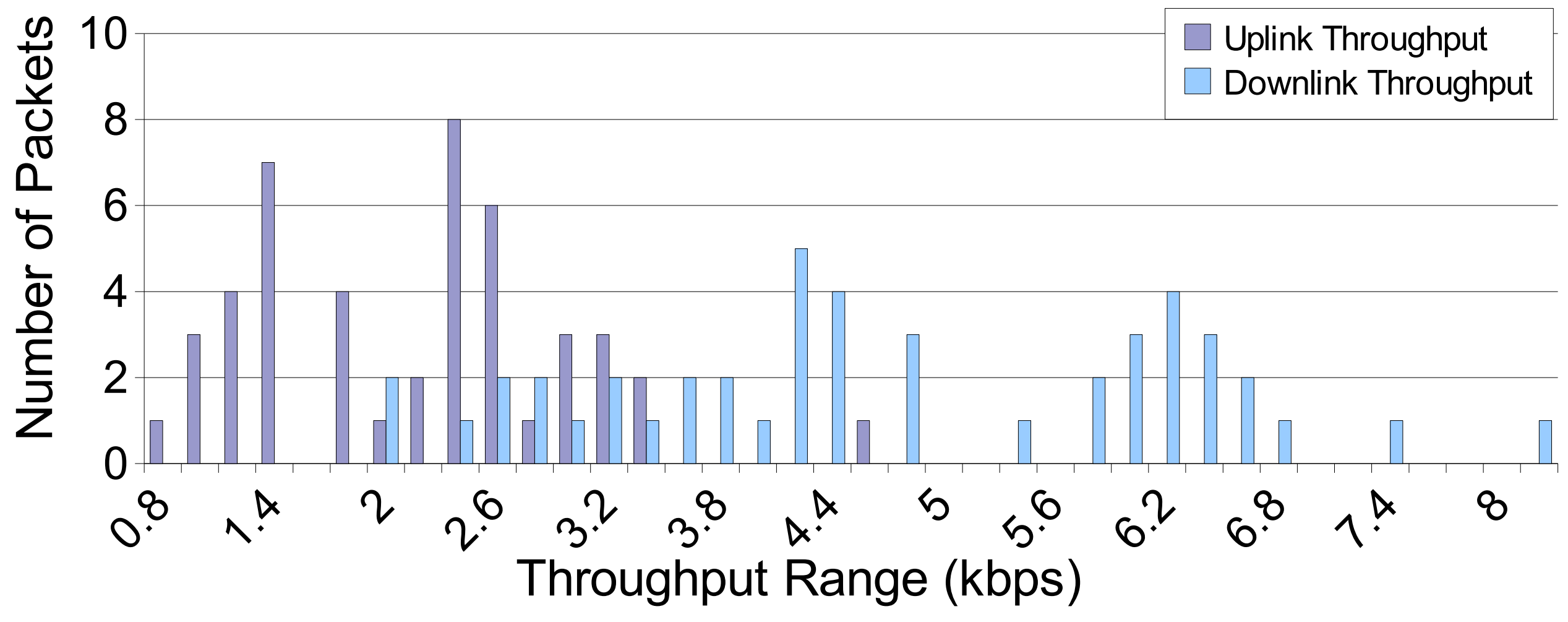




\section{Timeout Interval}

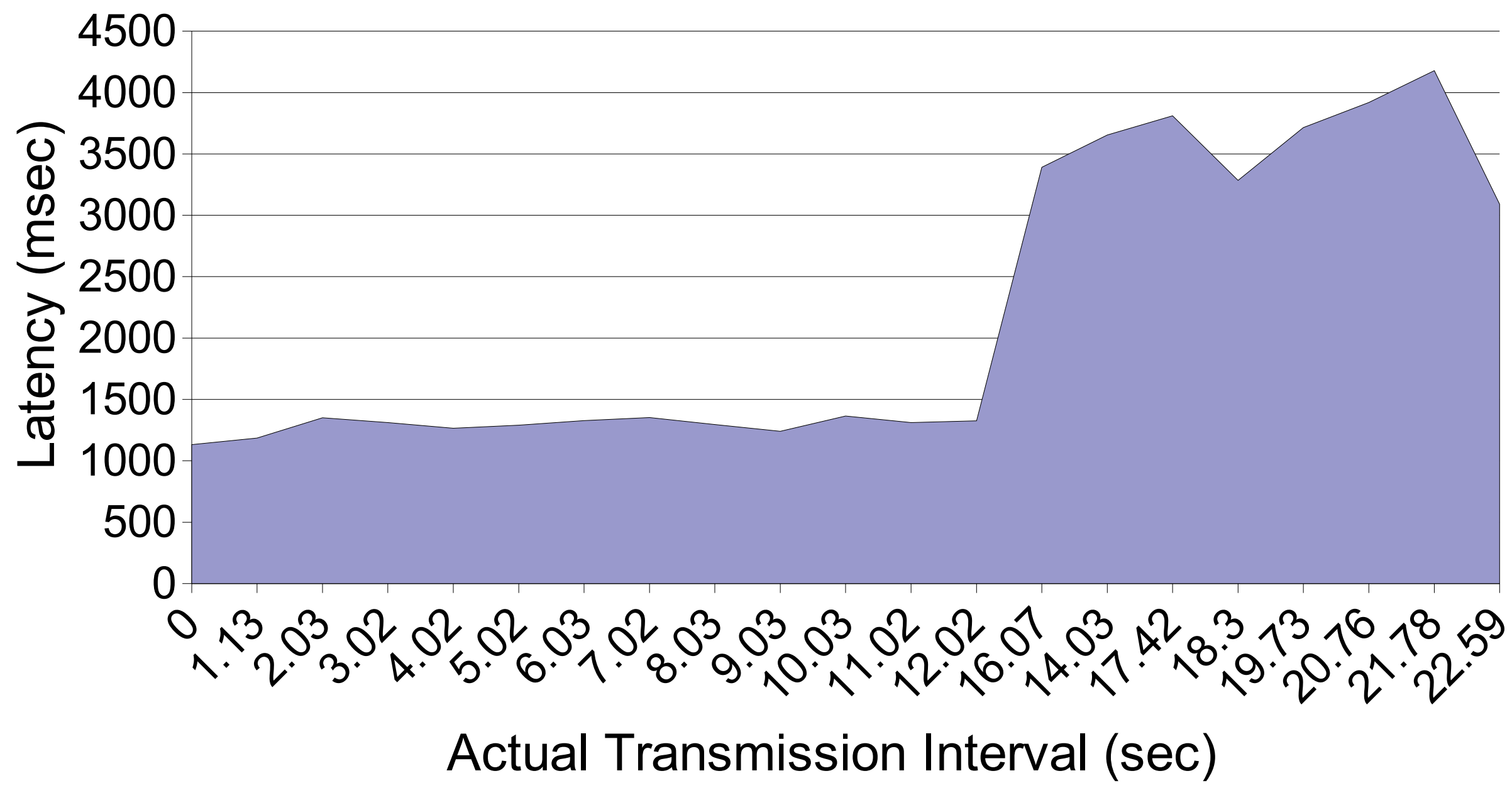




\section{Conclusions}

- Multiple device and service provider combinations

- Geographically distributed measurement

- Application emulation

- Better tools for data visualization

- Processing power, flash crowds, other 


\title{
Thank you
}

\author{
Mike P. Wittie \\ UC Santa Barbara \\ mwittie@cs.ucsb.edu
}

